\title{
Hyperuricaemia and gout
}

\author{
A Kopke, ${ }^{1}$ OBW Greeff ${ }^{2}$ \\ ${ }^{1}$ General Practitioner \\ ${ }^{2}$ Specialist Clinical Pharmacologist, Cytespace Africa, South Africa \\ Corresponding author, email: oppel.greeff@cytespace.co.za
}

Gout is a painful, inflammatory disease that affects more men than women. The incidence of gout has increased substantially over the past few decades, as evidenced by information from the Rochester project. Some of the risk factors for the development of gout include: increased ethanol intake, high dietary purine consumption, obesity and the use of certain drugs, such as diuretics. Another important risk factor for the development of gout is hyperuricaemia.

Hyperuricaemia results from an imbalance between the rate of production and excretion of uric acid in the body. An excess of uric acid thus builds up in the body, supersaturating body fluids and leading to the formation of monosodium urate crystals. These crystals accumulate in tissue and around joints, leading to an acute gout attack.

Gout can be divided into four phases, namely asymptomatic hyperuricaemia, acute gout attacks or recurrent gout, intercritical gout and chronic tophaceous gout. Various treatment options are available for gout, and the treatment for each gout patient is determined by the stage of the disease. Nonsteroidal anti-inflammatory drugs (NSAIDs), corticosteroids, corticotropin and colchicine are used for the treatment of acute gout attacks. Allopurinol and probenecid are used for long-term hypouricaemic therapy, while NSAIDs and colchicine are prescribed for the prophylaxis of future gout attacks.

All of these treatments have side-effects, ranging from mild to life-threatening in nature. There is a need for novel gout therapies that have fewer side-effects but are still as effective.

Keywords: hyperuricaemia, gout, gout treatment

\section{Introduction}

Gout was described by Hippocrates as "the disease of kings" due to its association with a rich diet. ${ }^{1}$ Risk factors for gout include high dietary purine consumption, e.g. various types of meat, seafood and certain vegetables, ethanol intake, obesity and the use of diuretics and low-dose aspirin., ${ }^{2,3}$ Gender is also a risk factor, due to the greater prevalence of gout in men than in women. ${ }^{1}$ The peak incidence of gout occurs between the ages of 30 and 50 years, and the prevalence continues to increase with age. ${ }^{4}$ However, in patients over the age of 60 , gout affects both men and women equally. ${ }^{5}$ Furthermore, postmenopausal women are more prone to develop gout, due to the possible effect of oestrogen increasing the renal clearance of uric acid. ${ }^{1}$ After the age of 80 , women are affected by gout more than men. ${ }^{5}$

The Western civilisation has had two major gout epidemics, one during the reign of the Roman Empire and the other during the British Empire. It has been said that we are currently in the middle of the third epidemic. ${ }^{2}$ The search for new gout treatments has increased considerably due to the large increase in the incidence of gout, as well as the renal and cardiovascular consequences of this disease. ${ }^{6}$

\section{Epidemiology}

The epidemiology of gout has been well documented in previous research. These studies indicate that a variety of factors may influence the development of gout, including hereditary, environmental and racial factors. ${ }^{1}$

\section{Incidence of gout in various populations}

The Normative Aging cohort study has provided useful information with regard to the incidence of gout in hyperuricaemic male patients. In patients who started off with a serum uric acid level of greater than $9.0 \mathrm{mg} / \mathrm{dL}$, the annual incidence of gout was $4.9 \%$. In the patients who initially had a serum uric acid level of between $7.0 \mathrm{mg} / \mathrm{dL}$ and $8.9 \mathrm{mg} / \mathrm{dL}$, the annual incidence rate decreased to $0.5 \%$, and this decreased further to $0.1 \%$ in patients who had an original serum uric acid level below $7.0 \mathrm{mg} / \mathrm{dL} .{ }^{7}$ This clearly indicates how important hyperuricaemia is as a risk factor for the development of gout.?

Furthermore, the Rochester Epidemiology Project has revealed that the incidence of gout increased from 45/100 000 between 1977 and 1978 to $63.2 / 100000$ in the period between 1995 and 1996. ${ }^{7}$

\section{Prevalence}

A $4.1 \%$ prevalence of hyperuricaemia was found in a previous study in people older than 75 years. ${ }^{2}$ However, care should be taken when comparing prevalence rates of gout in different studies, as there are so many variables to take into consideration. ${ }^{8}$ Examples of these variables include diagnostic criteria, sample size and even seasonal changes in levels of serum uric acid. ${ }^{8}$ 


\section{Pathophysiology}

Uric acid is formed from the breakdown of purines. ${ }^{1}$ Increased uric acid production may be caused by various factors, including nutritional, haematological and genetic factors. Other miscellaneous factors, such as obesity and excessive alcohol consumption, also increase urate production. ${ }^{1}$ While certain medications, such as some cytotoxic drugs, may increase uric acid levels in the body, other medications, such as cyclosporine, may decrease the renal excretion of uric acid. Various other renal, metabolic and genetic factors also decrease the excretion of uric acid. $^{1}$

Hyperuricaemia is caused by an imbalance in the rates of production and excretion of uric acid. This causes the body fluids to become supersaturated with uric acid, which means a level of 6.8 $\mathrm{mg} / \mathrm{dL} .{ }^{7}$ In men and postmenopausal women, hyperuricaemia is defined as a serum uric acid level of greater than $7.0 \mathrm{mg} / \mathrm{dL}$. In premenopausal women, hyperuricaemia is characterised by a serum uric acid level that is greater than $6.0 \mathrm{mg} / \mathrm{dL}{ }^{1}$

Hyperuricaemia causes an accumulation of monosodium urate crystals, which are deposited in the supersaturated body fluids and then are deposited in tissues and around joints during the asymptomatic hyperuricaemic gout stage. ${ }^{1,2,6}$ The monosodium urate crystals form tophi. ${ }^{1,2}$ The tophi cause inflammation, which leads to the symptoms of an acute gouty attack., ${ }^{2,6}$ The presence of tophi can lead to soft tissue damage and destruction of the afflicted joint, which can cause the deformities associated with gout. ' However, it is important to note that, while hyperuricaemia is a risk factor for gout, not all patients with hyperuricaemia develop gout. ${ }^{1}$ Many medicines prescribed for the treatment of gout patients specifically target hyperuricaemia.

Symptoms of an acute gout attack include pain, inflammation and erythema of the afflicted joint. ${ }^{9}$ The above-mentioned inflammation is caused by the release of various cytokines, including interleukin-1 $\beta$ (IL-1 $1 \beta$ ). IL-1 $\beta$ is an inflammatory cytokine that is associated with the leukocytosis and fever that often accompany acute gouty attacks. ${ }^{10}$ Both intracellular and extracellular signals activate the cryoprin (NLRP3) inflammasome, which in turn activates Caspase 1. Caspase 1 then cleaves pro-IL$1 \beta$ to form active IL-1 $\beta .^{10,11}$

As stated previously, current treatments for gout are aimed at decreasing levels of serum uric acid and treat one of the causes of gout. New research is currently under way to explore the potential use of an IL-1 $\beta$ blocker to treat gout. ${ }^{11}$

\section{Clinical phases of gout}

There are four recognised clinical stages in the development of gout, namely asymptomatic hyperuricaemia, followed by the acute gouty attack or recurrent gout, then the intercritical period and, lastly, chronic tophaceous gout. ${ }^{1}$

The first stage of gout, asymptomatic hyperuricaemia, may continue for many years without detection until the first gouty attack. In approximately half of gout patients, the first metatarsophalangeal joint is the first joint affected. ${ }^{2}$ Gouty attacks are initially infrequent, but, as the disease develops, the gouty attacks increase in frequency, duration and number of joints involved. ${ }^{2}$

The intercritical period of gout occurs after the clear-up of the first gouty attack. This is the stage when the affected joints appear to return to normal, although it is followed by the chronic gouty arthritis stage, during which there is such a large accumulation of monosodium urate crystals that the joints show evidence of this even between attacks. ${ }^{2}$

\section{Asymptomatic hyperuricaemia}

Asymptomatic hyperuricaemia appears to have adverse cardiovascular and/or renal effects. Is it possible that serum uratelowering therapy could decrease these adverse effects? The LIFE study, in which Losartan decreased adverse cardiovascular outcomes, is perhaps an example of this. Losartan is an angiotensin receptor blocker, but the authors of the study attributed the decreased adverse cardiovascular outcomes to Losartan's urate-lowering properties. Numerous risks are associated with the prophylactic treatment of asymptomatic hyperuricaemia, including the induction of gout flares, but there is also the possibility that prophylactic treatment at this stage would prevent progression to acute/recurrent gout. ${ }^{12}$ However, the use of prophylactic treatment for asymptomatic hyperuricaemia has been discouraged by numerous authors. Lifestyle modification is recommended instead. . $^{13,14}$

\section{Acute gouty attack or recurrent gout}

An acute gout flare is the result of a large inflammatory response to the deposition of the monosodium urate crystals. ${ }^{1}$ An acute attack of gout is characterised by pain, swelling and erythema in the affected joint. NSAIDs are considered to be the first-line treatment for acute or recurrent gout. ${ }^{9}$ The symptoms of an acute gout attack tend to be most pronounced eight to twelve hours after the start of the attack.?

The monosodium urate crystals that gather in hyperuricaemic patients activate both the humoral and cellular components of the immune system. These crystals stimulate the recruitment and consequent adhesion of neutrophils, leading to inflammation. The crystals activate various intracellular receptors, leading to the activation of various inflammasomes and bringing about increased production of IL-1 $\beta$, as demonstrated in Figure $1 .^{7}$

\section{Intercritical gout}

In a study of the uricosuric drug, probenecid, it was found that $70 \%$ of intercritical gout patients with a serum urate level of above $9 \mathrm{mg} / \mathrm{dL}$ had one or more gouty attacks in one year. Studies performed with the drugs allopurinol, benzbromarone and febuxostat have all shown that lowering serum urate levels to below $6 \mathrm{mg} / \mathrm{dL}$ decreases gouty attacks, depletes monosodium urate crystals in the joints, inhibits the formation of new tophi and decreases the size of existing tophi. ${ }^{12}$ Thus it is apparent that urate-lowering therapy is necessary in order to prevent longterm joint destruction in gout patients. 


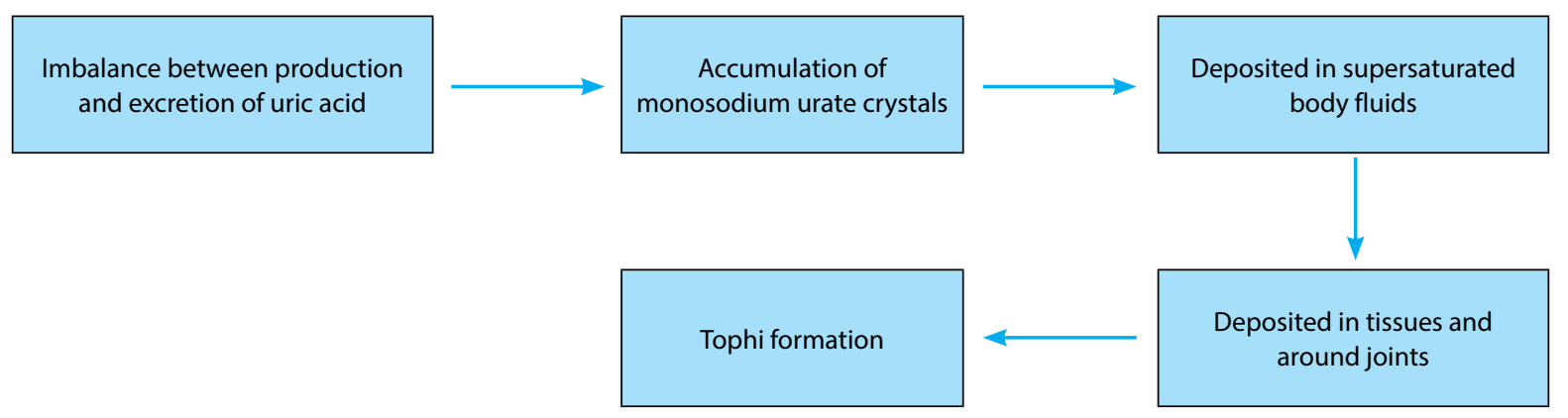

Figure 1: Formation of tophi

\section{Chronic tophaceous gout}

In chronic tophaceous gout, the symptoms continue between attacks, leading to significant pain and discomfort.' Uratelowering therapy, either a xanthine oxidase inhibitor or a uricosuric, is thus almost always indicated. This therapy will decrease the excess uric acid in the gout patient, consequently reducing the size of the tophi. In serious cases, surgical excision of the tophi may be performed. A serum urate level of $6.0 \mathrm{mg} / \mathrm{dL}$ or less has proved to be an important target level with regard to the treatment of chronic tophaceous gout. ${ }^{12}$

\section{Complications}

Complications may arise in gout due to the tophi that form from the accumulation of monosodium urate crystals in the affected joints. These tophi can cause damage to the surrounding joints and tissues, leading to chronic pain, and may even lead to deformities. ${ }^{1}$ Various renal complications may also occur. These complications include, but are not limited to, kidney stones, uric acid calculi and acute and chronic uric acid nephropathy. ${ }^{1}$ These complications are not only caused by the gout itself, but also by diseases that often accompany gout, such as hypertension, hyperlipidaemia, heart disease and type 2 diabetes. ${ }^{8}$ Furthermore, it is important to note that the drugs that the patients are prescribed to treat gout may bring about further complications, as discussed in the treatment section.

\section{Diagnosis}

An accurate diagnosis of gout forms an important part in accurately assessing and interpreting the results of clinical trials. An incorrect diagnosis would lead to an incorrect interpretation of the results. ${ }^{12}$

Gout may be diagnosed through the demonstration of an effusion of monosodium urate crystals in the affected tissue or joint. If this procedure is not possible to perform, gout may also be diagnosed by means of a review of medical history or the observation of any two of the following: podagra, tophus, one abrupt attack of monoarthritis and/or a response to colchicine within 48 hours. $^{8}$

The diagnosis of gout can also be made by ultrasound. The ultrasound has the ability to diagnose gout between acute attacks and even in patients with asymptomatic hyperuricaemia. ${ }^{2}$ Furthermore, ultrasound allows for the inspection of tophaceous deposits without the need for painful needle aspirations. As such, this technique is gaining in importance in the diagnosis of gout. ${ }^{2}$ Physicians also use the criteria developed by the American College of Rheumatology to confirm or dismiss a diagnosis of gout. $^{3}$

\section{Treatment}

Treatment is based on the particular clinical phase of gout that the patient is experiencing. Treatment for acute gout is aimed at reducing the pain and inflammation that accompany acute gout attacks, whereas treatment for the intercritical period of gout aims to maintain low levels of serum uric acid in order to prevent the formation of tophi. Chronic tophaceous gout is treated by initiating long-term hypouricaemic therapy. There currently is no evidence for the efficacy of treatment of asymptomatic hyperuricaemia. ${ }^{7}$

Treatment can also be divided into three groups, based on the purpose of the treatment. One group of drugs is intended to treat acute gout and the attacks that occur during this phase. The second group of drugs is used for the prevention of recurrences of gout, while the third group of drugs is aimed at lowering serum uric acid. ${ }^{2}$

\section{NSAIDs}

NSAIDs are considered to be the drugs of choice to treat acute gout. ${ }^{9}$ NSAIDs are also used to prevent the occurrence of future attacks. ${ }^{2}$ In the treatment of an acute attack, high doses of NSAIDs are prescribed during the first three to four days of an attack. Thereafter standard doses are prescribed for maintenance. ${ }^{7}$

In a study comparing the use of rofecoxib, diclofenac sodium and meloxicam in the treatment of acute gout, rofecoxib was found to be the most effective of all three NSAIDs, displaying a rapid onset of pain relief after dosing, with all three of the drugs giving similar numbers of side effects. ${ }^{9}$

Etoricoxib and lumiracoxib are two cyclo-oxygenase 2-selective inhibitors that have the same efficacy as NSAIDs in the treatment of acute gout. Two individual studies have suggested that the above two drugs may be of use in the treatment of acute gout in patients in whom NSAIDs are contraindicated.?

Although rofecoxib and lumiracoxib have since been removed from the market due to unwanted drug effects, there are still other cyclo-oxygenase inhibitors available, including the previously mentioned etoricoxib and meloxicam. ${ }^{7,9,15}$ 
Etoricoxib, in particular, has shown comparable efficacy to indomethacin in an acute gout clinical trial. ${ }^{12}$ Indomethacin is another NSAID used in acute gout, as well as in gout prophylaxis. ${ }^{1}$

One of the major adverse effects associated with NSAIDs is gastrointestinal damage. ${ }^{16}$ A proton pump inhibitor can be prescribed for gout patients on NSAIDs who face potential gastrointestinal risks. ${ }^{12}$ NSAIDs can also cause hypertension, fluid retention, congestive heart failure and central nervous system effects, in addition to the common gastrointestinal sideeffects. ${ }^{1,2}$ All of these side-effects are important to note due to the many comorbidities in the typical gout patient. ${ }^{2}$

As such, NSAIDs are often completely contraindicated in the elderly, where many comorbidities exist, in patients using warfarin, as well as in patients with cardiac, renal and hepatic diseases. ${ }^{7}$ Other treatments for acute gout include corticotrophin, corticosteroids and colchicine. ${ }^{1}$ The above-mentioned drugs are generally effective in the treatment of acute gout, although they are associated with numerous side-effects. ${ }^{1}$

As there is such a large variety of drugs that can be used for an acute gout attack, therapy is determined according to the stage of the attack, the severity of the attack and any existing comorbidities in the patient that may interfere with the therapy. ${ }^{12}$

\section{Colchicine}

Colchicine, an alkaloid found in certain plant extracts, has been used for many years in the treatment of gout and is effective in relieving the pain brought on by an acute gout attack. ${ }^{1,2}$ However, colchicine has a very small therapeutic index and as such can easily cause the gastrointestinal side-effects associated with colchicine, namely nausea, cramping and diarrhoea. Colchicine accumulates in patients with renal insufficiency, and as such is only recommended in patients with mild to moderate renal failure. ${ }^{2}$ Long-term side-effects of colchicine include neuropathy, neutropenia and vacuolar myopathy. ${ }^{7}$ These long-term sideeffects require monitoring.

Both NSAIDs and colchicine can be used for the prophylaxis of gout. Colchicine is recommended over NSAIDs in gout patients with renal failure and is also used as prophylaxis in the treatment of gout. ${ }^{2}$

The normal, low dose of colchicine is $1.2 \mathrm{mg}$ stat and the $0.6 \mathrm{mg}$ after $1 \mathrm{hr}$ - total dose of $1.8 \mathrm{mg}$. The high dose is $1.2 \mathrm{mg}$ stat, then $0.6 \mathrm{mg}$ hourly for 6 doses or until diarrhea occurs - thus a total dose of $4.8 \mathrm{mg}$

\section{Corticosteroids}

Corticosteroids have been found to be effective in the treatment of acute gout, whether they are administered intra-articularly, systemically or orally. ${ }^{2}$ They are indicated for use in gout patients who cannot tolerate, or have contraindications for the use of NSAIDs and colchicine. ${ }^{1}$ The concern with the use of corticosteroids are the many long-term side-effects of the drugs. ${ }^{1,17}$ Some of these side-effects include Cushing's syndrome, diabetes mellitus, hypertension and osteoporosis. ${ }^{17}$
However, as demonstrated in a clinical trial comparing the use of oral indomethacin and paracetamol to the use of oral prednisolone and paracetamol, prednisolone (corticosteroid) proved to be just as effective as indomethacin (NSAID) with regard to the treatment of acute gout, and was also associated with fewer adverse effects than indomethacin. As such, it appears that the short-term use of a corticosteroid, such as prednisolone, can be effective in the treatment of acute gout without the risks of the long-term side-effects of corticosteroids. ${ }^{17}$

\section{Corticotropin}

Corticotropin, or ACTH as it is commonly known, has been used in the treatment of acute gout for many years. In one clinical trial in which indomethacin was compared to the use of ACTH in acute gout, ACTH brought about faster pain relief than indomethacin, and the ACTH treatment group had far fewer side-effects than the indomethacin group. ${ }^{1}$

Corticotropin is used as a treatment for polyarticular flares in acute gout when the first-line therapies have either failed or are contraindicated. ${ }^{1,7}$ When ACTH is used in conjunction with colchicine, it is effective in the treatment of acute gout and causes less side-effects than indomethacin. ${ }^{1}$ The side-effects of colchicine and ACTH combination therapy include fluid retention, poor control of glucose levels in diabetes, hypokalaemia and the relapse of gout or rebound arthritis. ${ }^{1,7}$

\section{Allopurinol}

Allopurinol is not used in asymptomatic hyperuricaemia but is indicated in long-term hypouricaemic therapy. Allopurinol is a xanthine oxidase inhibitor that improves gout by decreasing the amount of serum uric acid through the inhibition of the xanthine oxidase enzyme, which is necessary to produce uric acid. ${ }^{2}$ The normal dose of allopurinol is $100 \mathrm{mg}$ TDS or $300 \mathrm{mg}$ nocte. Often this dose will not result in a serum uric acid of $<6 \mathrm{mg} / \mathrm{dL}$ and it is recommended that the dose can be increased to a maximum of $900 \mathrm{mg} /$ day.

Allopurinol is considered to be very effective in terms of longterm hypouricaemic therapy, and is recommended for use in patients with high serum uric acid levels, nephrolithiasis and renal impairment. ${ }^{2}$ Serum urate levels need to be monitored continuously in order to maintain the appropriate dosage of allopurinol. Elderly patients, as well as patients with impaired kidney function, are generally prescribed lower doses of allopurinol. ${ }^{7}$

Although allopurinol is effective, various side-effects have been noted. These side-effects vary in degree of severity, for instance some patients complain of only mild gastrointestinal side-effects. On the other end of the scale is the life-threatening "allopurinol hypersensitivity syndrome", which is characterised by fever, rash, eosinophilia, hepatitis and renal failure. ${ }^{2,7}$ Risk factors for the development of allopurinol hypersensitivity syndrome include allopurinol therapy initiation, renal insufficiency and the use of diuretics. $^{7}$ 
Allopurinol also has dangerous drug interactions, for example with azathioprine. Various allergic reactions to allopurinol have also been reported, thus patients on allopurinol require monitoring for possible adverse events. ${ }^{2}$

Probenecid is also used for long-term hypouricaemic therapy. It is a uricosuric drug and, as such, lowers serum uric acid by increasing the amount of urinary excretion of uric acid. ${ }^{1,2}$ Probenecid also requires two daily doses, which may affect compliance. While probenecid does have interactions with certain drugs and is considered to be less effective than allopurinol, it is sometimes the drug of choice for long-term hypouricaemic therapy due to its far less risky side-effect profile in comparison to allopurinol. ${ }^{2}$

Other uricosuric drugs that may be used for hypouricaemic therapy include benzbromarone, sulfinpyrazone, losartan and fenofibrate. ${ }^{7}$ These uricosuric drugs are not widely used in practice because of their many drug interactions, their loss of effectiveness in patients with low glomerular filtration rates, and their possible role in promoting nephrolithiasis.?

New drugs for the treatment of gout include febuxostat and uricase. Febuxostat proved to be an effective hypouricaemic therapy in a clinical trial comparing the use of febuxostat and allopurinol, but febuxostat, upon initiation of therapy, induced acute gout flares in up to $70 \%$ of patients. Furthermore, there are concerns about the long-term safety of febuxostat with regard to hepatic and cardiovascular adverse events. ${ }^{7}$ The normal dose of febuxostat is $40-60 \mathrm{mg}$ daily.

Uricase, namely polyethylene glycol-linked uricase, is being investigated in Phase 3 clinical trials in order to determine whether it is effective in controlling hyperuricaemic patients with chronic gout, and patients who have not responded to conventional therapy. ${ }^{6,7}$ Long-term hypouricaemic therapy is intended to decrease levels of serum uric acid in order to decrease acute gout attacks, while at the same time preventing damage over time to the patient's joints by decreasing the size and amount of tophi. ${ }^{2}$

\section{Diet and lifestyle}

In addition to the medical intervention necessary in gout, patients are encouraged to make certain diet and lifestyle changes. The aim is to reduce obesity, decrease the intake of purine-rich foods, such as various types of meat, seafood and even certain vegetables, and decrease excessive alcohol consumption. ${ }^{1,3}$ Examples of purine-rich foods include animal liver, beef, tuna, peas and lentils. ${ }^{3}$ Lifestyle modification would involve a drastic change in the patient's diet, cutting out many of the purine-rich foods. ${ }^{1}$ It has also been suggested that an increased intake of dairy products may decrease the incidence of gout. ${ }^{3}$ While the various drugs available for the treatment of gout clearly play a vital role, it is important to note that diet and lifestyle modifications also form an essential part of the treatment plan. Furthermore, diet and lifestyle modification have the dual benefit of not only improving gout symptoms, but also many of the diseases associated with gout, such as hypertension, hyperlipidaemia and diabetes mellitus. ${ }^{8}$
A practical approach to the treatment of an acute attack of gout:

1. High doses of a NSAID/COX-2 inhibitor, e.g. diclofenac $50 \mathrm{mg}$ TDS PC.

2. Prednisone $15 \mathrm{mg}$ Q8H - 4 doses.

3. Increase fluid intake: $>2 \mathrm{~L}$ of urine excretion daily.

4. Alkalinise urine: CitroSoda BD. Do not drink/eat anything that can make the urine acid, e.g. orange juice, tomatoes etc.

5. Avoid drugs that decrease the excretion of uric acid, e.g. low doses asprin.

\section{Conclusion}

Hyperuricaemia, one of the major risk factors for the development of gout, is caused by an imbalance between the rates of production and excretion of uric acid. An excess of uric acid thus supersaturates the body fluids, leading to the deposition of monosodium urate crystals in tissues and joints, which in turn leads to the initiation of an acute attack of gout.

Gout is a painful, occasionally debilitating disease and is divided into four clinical phases, namely asymptomatic hyperuricaemia, acute gouty attack or recurrent gout, intercritical gout and chronic tophaceous gout. Each of these phases has various possible treatments. NSAIDs, colchicine, corticosteroids and corticotrophin are used for the treatment of acute gout attacks in order to relieve pain. NSAIDs and colchicine are prescribed for the prophylaxis of gout. Allopurinol, probenecid and other uricosurics are used in long-term hypouricaemic therapy in order to decrease serum uric acid levels and to prevent long-term damage to joints.

Due to the untoward and, in some cases, potentially lifethreatening side-effects of the various gout therapies, there most definitely is a need for novel therapies to treat gout. Detailed patient evaluation forms the basis of a clinician's decision on the choice of therapy for gout patients. As such, more attention needs to be given to patient evaluation in order to personalise gout therapy for every patient. Emphasis should also be placed on the importance of diet and lifestyle modifications in addition to gout therapy so as to prevent gout and acute gout attacks.

\section{References}

1. Kim KY, Schumacher HR, Hunsche E, Wertheimer Al, Kong SX. A literature review of the epidemiology and treatment of acute gout. Clin Ther. 2003;25(6):1593617. https://doi.org/10.1016/S0149-2918(03)80158-3.

2. Falasca GF. Metabolic diseases: gout. Clin Dermatol. 2006;24:498-508. https://doi. org/10.1016/j.clindermatol.2006.07.015.

3. Choi HK, Atkinson K, Karlson EW, Willett W, Curhan G. Purine-rich foods, dairy and protein intake, and the risk of gout in men. N Engl J Med. 2004 March 11;350(11):1093-103. https://doi.org/10.1056/NEJMoa035700.

4. Jacobs CL, Stern PJ. An unusual case of gout in the wrist: the importance of monitoring medication dosage and interaction. A case report. Chiropr Osteopat. 2007 October 9;15(16). https://doi.org/10.1186/1746-1340-15-16.

5. Kirby BS, McTigue JC, Edwards NL. Pain management in gout. Curr Pain Headache Rep. 2008 December;12(6):418-22. https://doi.org/10.1007/ s11916-008-0071-9.

6. Sherman MR, Saifer MGP, Perez-Ruiz F. PEG-uricase in the management of treatment-resistant gout and hyperuricemia. Adv Drug Deliv Rev. 2008;60:59-68. https://doi.org/10.1016/j.addr.2007.06.011.

7. Gaffo AL, Saag KG. Management of hyperuricemia and gout in CKD. Am J Kidney Dis. 2008;52(5):994-1009. https://doi.org/10.1053/j.ajkd.2008.07.035. 
8. Darmawan J, Lutalo SK. Gout and hyperuricaemia. Bailliére's Clin Rheumatol. 1995 February;9(1):83-94. https://doi.org/10.1016/S0950-3579(05)80146-3.

9. Cheng $\mathrm{T}$, Lai $\mathrm{H}$, Chiu $\mathrm{C}$, Chen $\mathrm{Y}$. A single-blind, randomized, controlled trial to assess the efficacy and tolerability of rofecoxib, diclofenac sodium, and meloxicam in patients with acute gouty arthritis. Clin Ther. 2004;26(3):399-406. https://doi.org/10.1016/S0149-2918(04)90035-5.

10. Ren $K$, Torres $R$. Role of interleukin- $1 \beta$ during pain and inflammation. Brain Res Rev. 2009;60(1):57-64. https://doi.org/10.1016/j.brainresrev.2008.12.020.

11. Hoffman HM, Yasothan U, Kirkpatrick P. Rilonacept. Nat Rev Drug Discov. 2008;7:385-6. https://doi.org/10.1038/nrd2579.

12. Chen LX, Schumacher HR. Gout: can we create and evidence-based systematic approach to diagnosis and management? Best Pract Res Clin Rheumatol. 2006;20(4):673-84. https://doi.org/10.1016/j.berh.2006.05.006.
13. Richardson RMA. Treatment of asymptomatic hyperuricemia: When do you have a good reason to provide therapy? Can Fam Physician. 1991 February;37:459-63.

14. Fujimori S. [Establishment of therapeutic goal and plan of gout and asymptomatic hyperuricemia.] Nippon Rinsho. 2008 Apr;66(4):729-35.

15. Hinz B, Brune K. Can drug removals involving cyclooxygenase-2 inhibitors be avoided? A plea for human pharmacology. Trends Pharmacol Sci. 2008 August;29(8):391-397. https://doi.org/10.1016/j.tips.2008.06.004.

16. Del Favero A. Anti-inflammatory and antipyretic analgesics and drugs used in gout. In: Aronson JK, editor. Side effects of drugs. Elsevier; 2008. p. 25-36. https:// doi.org/10.1016/S0378-6080(08)00009-3.

17. Man CY, Cheung ITF, Cameron PA, Rainer TH. Comparison of oral prednisolone/ paracetamol and oral indomethacin/paracetamol combination therapy in the treatment of acute goutlike arthritis: A doubleblind, randomized, controlled trial. Ann Emerg Med. 2007 May;49(5):670-7. https://doi.org/10.1016/j. annemergmed.2006.11.014. 